\title{
Design for High-Power Single-Mode Operation From 2-D Surface-Emitting ROW-DFB Lasers
}

\author{
Shuang Li and Dan Botez
}

\begin{abstract}
Stable single-mode single-lobe operation to high powers is predicted for two-dimensional surface-emitting lasers, if second-order distributed feedback/distributed Bragg reflector (DFB/DBR) gratings are preferentially placed in the elements of a resonant-optical-waveguide array. Beside their usual functions (i.e., feedback and outcoupling), the gratings act as an effective array-mode selector due to different interaction with the gratings of different array modes. The in-phase array mode is strongly favored to lase around its (lateral) resonance due to better field overlap with DFB region and lower interelement absorption loss than for nonresonant array modes. For 20-element arrays with $700 / 600 \mu \mathrm{m}$ DFB/DBR gratings, emitting at $\lambda=0.98 \mu \mathrm{m}$, high $\left(\sim 100 \mathrm{~A} / \mathrm{cm}^{2}\right)$ intermodal discrimination $\Delta J_{\mathrm{th}}$ is obtained. $\Delta J_{\mathrm{th}}$ is enhanced to $\sim 225 \mathrm{~A} / \mathrm{cm}^{2}$ by introducing free-carrier absorption in the array-interelement regions.
\end{abstract}

Index Terms-Active photonic crystals, distributed-feedback (DFB) lasers, phase-locked oscillators, semiconductor lasers, surface-emitting lasers.

$\mathbf{T}$ WO-DIMENSIONAL (2-D) single-mode single-lobe grating surface emitters (GSE) are ideal high-power $(\geq 1 \mathrm{~W})$ coherent sources due both to their monolithic nature and, thus, intrinsic reliability (as opposed to external-cavity vertical-cavity surface-emitting lasers) as well as the potential for scaling the power via coherent coupling of the sources at the wafer level [1]. Three types of 2-D single-mode uniphase (i.e., emitting in a single lobe) surface emitters have been reported or proposed [2]-[4]. They involve angled gratings [2], a curved-grating unstable resonator [3], and hexagonal-lattice gratings [4]. However, none of these devices have strong built-in dielectric structures for lateral-optical-mode control and stability, and as a result are vulnerable to temperatureand carrier-induced dielectric-constant variations [2], [3]. A good example of such behavior is the unstable resonator device [3], which, while operating single-mode to high peak-pulsed powers in a single, off-normal beam, can become multimode in continuous-wave $(\mathrm{CW})$ operation due to thermal lensing.

Here, we present a 2-D GSE structure which combines the strong built-in index guiding $(\Delta n \geq 0.05)$ for lateral-mode control and stability of resonant-optical-waveguide (ROW) arrays [5], [6], with an inherently efficient single-lobe surface-emitting distributed-feedback (DFB)-type structure [7], [8]. The result is a device which holds the potential to achieve watt-range $\mathrm{CW}$ surface-emitted powers in a stable single mode.

Manuscript received July 12, 2004; revised October 10, 2004. This work was supported by the National Science Foundation (NSF) under Grant ECS0200321.

The authors are with the Reed Center for Photonics, University of Wisconsin, Madison, WI 53706 USA (e-mail: botez@engr.wisc.edu).

Digital Object Identifier 10.1109/LPT.2004.842388
ROW arrays, which are in effect laterally resonant active photonic crystals [6], have demonstrated diffraction-limited-beam operation to very high pulsed $(10 \mathrm{~W})$ and $\mathrm{CW}(1.6 \mathrm{~W})$ output powers due to their large lateral (effective) index steps and uniform lateral-field intensity profiles [6]. We have also shown that second-order DFB/distributed Bragg reflector (DBR) devices with central $\pi$ phaseshift can provide single-frequency single-lobe surface emission with high efficiency [7], [8]. The relatively uniform guided-field profile [in the active (DFB) region] makes the device invulnerable to gain spatial hole burning, thus ensuring stable operation to high drive levels above threshold. Recently, we demonstrated this concept with a ridge-guide for lateral-mode control [9]. Single-frequency lasing in an orthonormally emitted single-lobe diffraction-limited beam was obtained to $3 \times$ threshold from unoptimized devices.

We theoretically show that inserting DFB/DBR gratings with $\pi$ phaseshift only in the elements of a ROW array provides a 2-D GSE device with both large intermodal discrimination as well as large (lateral) index steps, thus ensuring single-frequency single-lobe emission to high $\mathrm{CW}$ power levels.

Fig. 1(a) depicts a three-dimensional schematic structure of the proposed device for emission at $\lambda=0.98 \mu \mathrm{m}$. An Al-free structure allows for easy regrowth of the interelement regions of the ROW array [Fig. 1(b)]. The laser base structure has a $1-\mu \mathrm{m}$-thick $n$-InGaP lower cladding layer. The optical confinement and gain are provided by a separate-confinement-heterostructure double-quantum-well structure consisting of $0.1-\mu$ m-thick InGaAsP $(1.6 \mathrm{eV})$ confinement layers surrounding two $70-\AA \AA$-thick InGaAs quantum wells. A 200- $\AA$-thick p-GaAs etch-stop layer is placed in the middle of a 400-nm-thick p-InGaP upper cladding layer to control the etching depth. The base is capped with $0.1-\mu \mathrm{m}$-thick $\mathrm{p}^{+}$-GaAs layer. Then a second-order grating with central $\pi$ phaseshift (not shown) is made in the $\mathrm{p}^{+}$-GaAs layer, in the longitudinal direction [7], [8]. The ROW-array structure is formed in the lateral direction by selective etching and regrowth: The $\mathrm{p}^{+}-\mathrm{GaAs}$ and $\mathrm{p}$-InGaP materials above the etch-stop layer are etched by using $\mathrm{SiO}_{2}$ stripes as a mask, and then the spaces left are refilled with high-index material (i.e., p-GaAs) to form the interelement regions of a ROW array [i.e., the high effective-index regions at the top of Fig. 1(b)]. As a result, the gratings are located only in the array-element regions (i.e., the low effective-index regions). $\mathrm{SiO}_{2}$ is then deposited and patterned to define the DFB and DBR regions. Au is deposited to form the electrical contact in the DFB region. To insure no back reflections into the lasing cavity, absorbing InGaAs regions are then regrown [3] at the ends of DBR regions and at the array edges. Light 


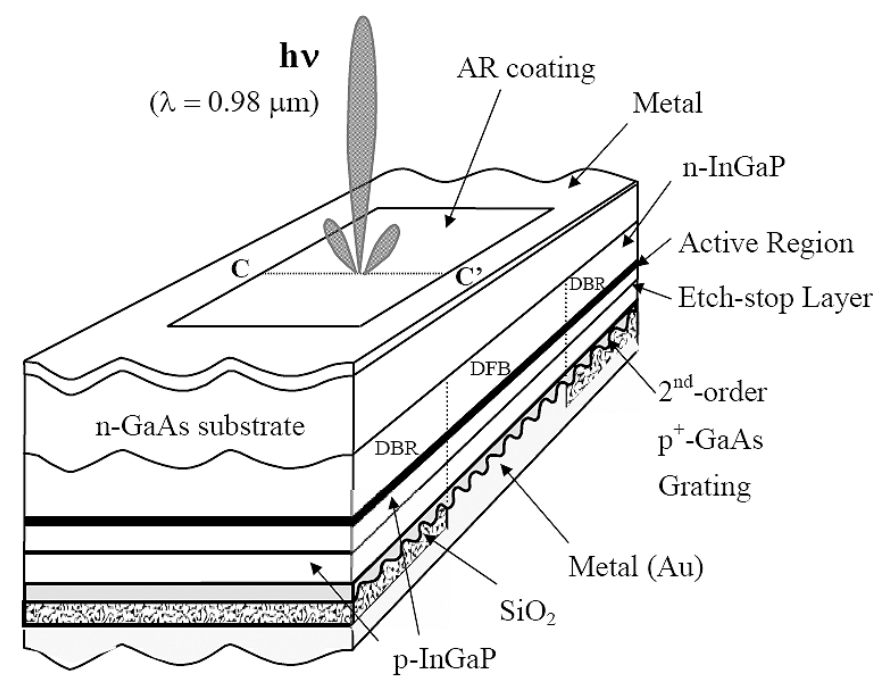

(a)

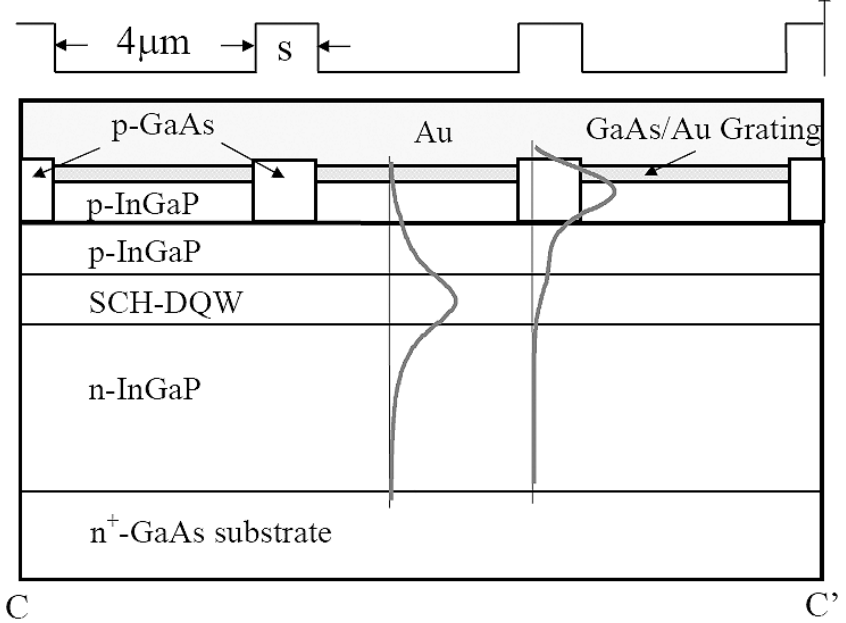

(b)

Fig. 1. (a) Schematic representation of 2-D surface emitter composed of an antiguided array and a second-order DFB/DBR grating structure with central $\pi$ phaseshift (not shown). (b) Device schematic cross section with transverse-field profiles in the element and interelement regions of the antiguided array (i.e., in the low- and high-effective-index regions).

is emitted through an antireflection-coated window in the substrate-side metallization. For an array with $4-\mu$ m-wide elements [Fig. 1(b)] and interelement-region width $s$ of $\sim 1 \mu \mathrm{m}$, 0.28 - $\mu$ m-thick regrown $\mathrm{p}$-GaAs regions give large $(\sim 0.055)$ effective-index steps at the in-phase mode (lateral) resonance.

As seen in Fig. 1(b), the interelement fields are "sucked up" into the high-index regrown $\mathrm{p}-\mathrm{GaAs}$ regions, thus experiencing strong losses in the p-metal (i.e., Au). Since the amount of (lateral) field in the element regions is strongly array-mode dependent [5], the array modes' interactions with the gratings are quite different. The array mode with the most field in the element regions experiences the strongest interaction with the gratings and the least interelement loss, and, as we will see, it is the one mode strongly favored to lase.

For a 20-element resonant array, we calculate [10] the amount of field intensity in all array elements $\Gamma_{\mathrm{el}}$ for the in-phase mode ( $L=38$ ) and other competing modes (adjacent modes $L=37$ and $L=39$, and the out-of-phase modes $L=19$ and $L=57$ ).
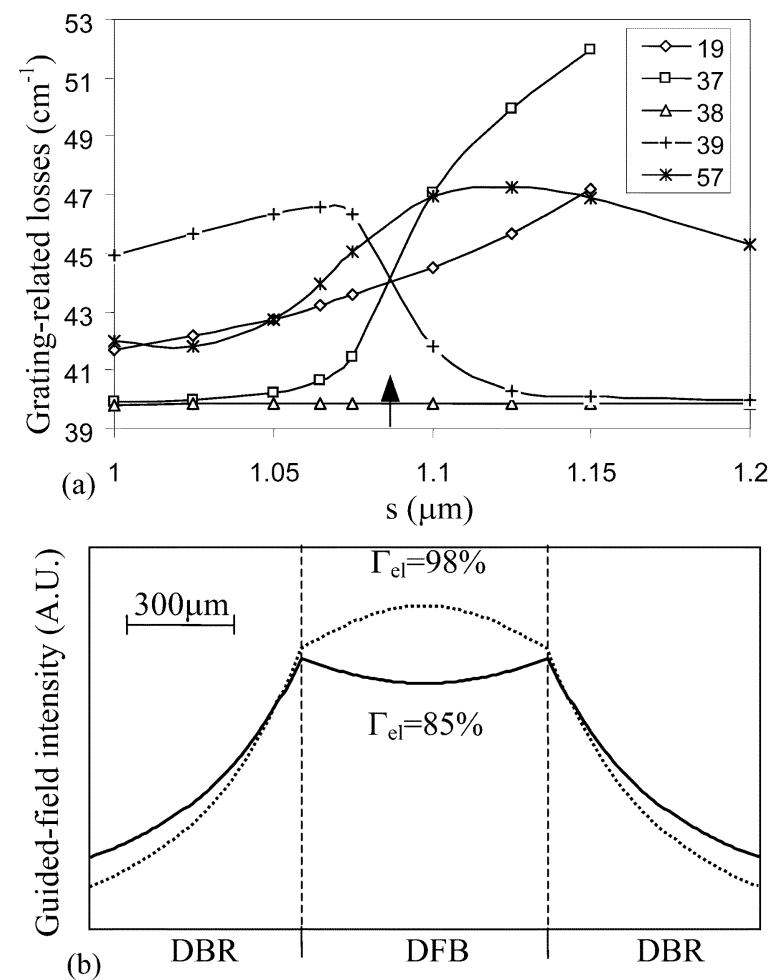

Fig. 2. (a) Grating-related losses for relevant array modes of a 20-element ROW array versus the interelement spacing. Mode 38 is the in-phase mode; Modes 37 and 39 are adjacent modes and Modes 19 and 57 are out-of-phase modes. (b) Guided-field longitudinal intensity profiles for array mode with $98 \%$ field in the elements (i.e., Mode 38) and 85\% field in the elements (i.e., Modes 19, 37 and 39).

For $4-\mu \mathrm{m}$-wide elements, at resonance (i.e., $s=1.09 \mu \mathrm{m}$ ), the desired in-phase mode has most of its field in the element regions $\left(\Gamma_{\mathrm{el}}=98.5 \%\right)$. The other competing modes, being nonresonant at that point, have smaller $\Gamma_{\mathrm{el}}$ values $(\leq 85 \%)$, as expected [10].

The coupling coefficients for the DFB and DBR regions are numerically calculated [8]. For different array modes, the effective coupling coefficients are different and scale linearly according to their amount of field intensity in the array elements (i.e., $\Gamma_{\mathrm{el}}$ ). The grating-related losses $2 \alpha$, which are the sum of the surface-radiation loss $\alpha_{\text {surf }}$ and DBR-grating-end losses $\alpha_{\text {end }}$ divided by the longitudinal field-intensity overlap with the DFB region $\Gamma_{\mathrm{lg}}$, can be obtained by solving the entire DFB/DBR structure by using the transfer-matrix method [8]. A typical result is shown in Fig. 2(a). At resonance, the difference in grating-related loss is $4.4 \mathrm{~cm}^{-1}$ between the in-phase mode (i.e., Mode 38 ) and the modes with $85 \% \Gamma_{\text {el }}$ value. This loss differential mainly reflects the difference in $\Gamma_{\lg }$ values between the in-phase mode and the $85 \%-\Gamma_{\text {el }}$ modes. For our devices $\Gamma_{\lg }$ is $59 \%$ for Mode 38 and 54\% for nonresonant Modes 19, 37, and 39. That is, the competing modes, seeing less of the gratings, have lower effective coupling coefficients; subsequently, they spread longitudinally and, thus, have lower $\Gamma_{\lg }$ values than the in-phase mode. As an example, we show in Fig. 2(b) the longitudinal-field distributions for array modes of different $\Gamma_{\mathrm{el}}$. A mode with $\Gamma_{\mathrm{el}}=85 \%$ spreads out longitudinally more and has larger field intensity at the ends of DBR regions than a mode with a $98 \% \Gamma_{\text {el }}$ value. Therefore, for our device, the 

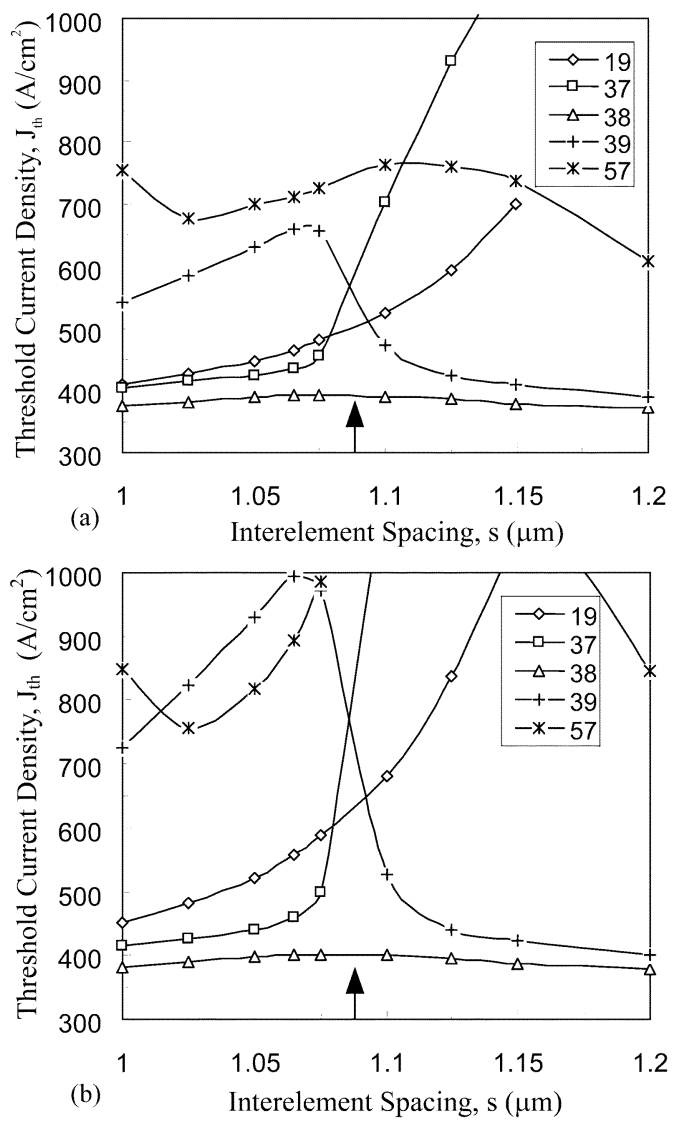

Fig. 3. Threshold-current densities of the array modes of a 20-element array with DFB/DBR gratings versus the interelement spacing. (a) No interelement free-carrier absorption loss. (b) Interelement loss due to absorption in heavily doped p-GaAs. Vertical arrows correspond to the in-phase mode resonance.

grating acts as a lateral-mode selector besides its two other roles, feedback and outcoupling.

To assess both longitudinal and lateral intermodal discrimination, one has to compute the threshold gain for each array mode. The DFB-region threshold gain [8] for the $m$ th array mode $g_{\mathrm{th}, m}$ is defined as the sum of the grating-related losses, $2 \alpha$, the array-related losses (i.e., edge-radiation loss and interelement loss due to $\mathrm{Au}, \alpha_{\mathrm{Au}}$, and/or free carriers, $\alpha_{\mathrm{fc}}$, and the internal cavity loss $\alpha_{i}$. Then, by using a relationship between the threshold-current density $J_{\text {th }}$ and the threshold gain, one can compute the $J_{\text {th }}$ values for each array mode

$$
J_{\mathrm{th}, m}=110 \frac{A}{\mathrm{~cm}^{2}} \times \exp \left[\frac{g_{\mathrm{th}, m}}{\left(\Gamma_{2 \mathrm{D}, m} \cdot 1130 \mathrm{~cm}^{-1}\right)}\right]
$$

where $\Gamma_{2 \mathrm{D}, m}$ is the 2-D optical-confinement factor [10].

Threshold-current densities for different array modes $J_{\mathrm{th}, m}$ were computed as a function of the interelement width $s$ [Fig. 3(a)]. The device has a 20-element array and gratings composed of a $700-\mu \mathrm{m}$-long DFB region and $600-\mu \mathrm{m}$-long DBR regions. At its resonance, the in-phase mode has a $J_{\text {th }}$ value of around $390 \mathrm{~A} / \mathrm{cm}^{2}$ and an intermodal discrimination $\Delta J_{\text {th }}$ of about $100 \mathrm{~A} / \mathrm{cm}^{2} . \alpha_{\mathrm{Au}}$ is $4.1 \mathrm{~cm}^{-1}$ for the nonresonant modes. Over the 1.07-1.11- $\mu \mathrm{m}$ range in $s, \Delta J_{\text {th }}$ is larger than
$60 \mathrm{~A} / \mathrm{cm}^{2}$ and it is due $\sim 50 \%$ to $\alpha_{\mathrm{Au}}$ and $\sim 50 \%$ to differences in $\Gamma_{\mathrm{lg}}$. The beam pattern for this design has a beam aspect ratio of 10 (i.e., $0.06^{\circ} \times 0.6^{\circ}$ full-width at half-maximum), yet using 40-element arrays [6], the aspect ratio can be lowered to five, which allows for easy circularization.

Further discrimination can be obtained by introducing (nonsaturable) free-carrier absorption $\alpha_{\mathrm{fc}}$ in the interelement regions. That can be achieved by heavily doping only the top part of the regrown p-GaAs region. For the case of heavily doping $\left(p=10^{20} \mathrm{~cm}^{-3}\right)$ the top $0.1 \mu \mathrm{m}$ of the p-GaAs interelement material $\left(\alpha_{\mathrm{fc}} \sim 600 \mathrm{~cm}^{-1}\right)$, the in-phase-mode $J_{\mathrm{th}}$ value remains basically the same $\left(\sim 400 \mathrm{~A} / \mathrm{cm}^{2}\right)$, while $\Delta J_{\text {th }}$ at resonance jumps from 100 to $225 \mathrm{~A} / \mathrm{cm}^{2}$ [Fig. 3(b)]. Similarly, $\Delta J_{\text {th }}$ over the $1.07-1.11-\mu \mathrm{m}$ range in $s$ increases from $\sim 60$ to $\sim 100 \mathrm{~A} / \mathrm{cm}^{2}$. Thus, by introducing additional interelement loss, the fabrication tolerances are significantly loosened which leads to higher yield of single-mode devices. This large amount of intermodal discrimination coupled with the device's high degree of guided-field uniformity in two dimensions should allow for stable single-mode $\mathrm{CW}$ operation to high powers. Calculated slope efficiencies of $0.78 \mathrm{~W} / \mathrm{A}$ lead to estimated single-mode $\mathrm{CW}$ powers of at least $2 \mathrm{~W}$.

\section{ACKNOWLEDGMENT}

The authors gratefully acknowledge valuable discussions with S. Macomber.

\section{REFERENCES}

[1] L. J. Mawst, D. Botez, M. Jansen, C. Zmudzinski, S. Ou, M. Sergant, C. $\mathrm{Tu}, \mathrm{T}$. J. Roth, G. Peterson, M. Valley, and J. J. Yang, "Two-dimensional surface-emitting leaky-wave coupled laser arrays," IEEE J. Quantum Electron., vol. 29, no. 6, pp. 1906-1917, Jun. 1993.

[2] K. M. Dzurko, A. Hardy, R. J. Lang, D. R. Scifres, D. F. Welch, and R. G. Waarts, "Distributed Bragg reflector ring oscillators: A large aperture source of high single-mode optical power," IEEE J. Quantum Electron., vol. 29, no. 6, pp. 1895-1899, Jun. 1993.

[3] S. H. Macomber, J. S. Mott, B. D. Schawrtz, R. S. Setzko, J. J. Power, P. A. Lee, D. P. Kwo, R. M. Dixon, and J. E. Logue, "Curved-grating, surface-emitting DFB lasers and arrays," Proc. SPIE, vol. 3001, pp. 42-54, 1997.

[4] I. Vurgaftman and J. R. Meyer, "Design optimization for high-brightness surface-emitting photonic-crystal distributed-feedback lasers," IEEE J. Quantum Electron., vol. 39, no. 6, pp. 689-700, Jun. 2003.

[5] D. Botez, "High-power monolithic phase-locked arrays of antiguided semiconductor diode lasers," in Proc. Inst. Elect. Eng., vol. 139, Feb. 1992, pp. 14-23.

[6] - "High-Power monolithic single-mode diode lasers employing active photonic lattices," Proc. SPIE, vol. 4993, pp. 20-27, 2003.

[7] G. Witjaksono and D. Botez, "Surface-emitting, single-lobe operation from second-order distributed-reflector lasers with central grating phaseshift," Appl. Phys. Lett., vol. 78, pp. 4088-4090, 2001.

[8] S. Li, G. Witjaksono, S. H. Macomber, and D. Botez, "Analysis of surface-emitting second-order distributed feedback lasers with central grating phaseshift," IEEE J. Sel. Topics Quantum Electron., vol. 9, no. 5, pp. 1153-1165, Sep./Oct. 2003.

[9] G. Witjaksono, S. Li, J. J. Lee, D. Botez, and W. K. Chan, "Single-lobe, surface-normal beam surface emission from second-order distributed feedback lasers with half-wave grating phaseshift," Appl. Phys. Lett., vol. 83, pp. 5365-5367, Dec. 2003.

[10] D. Botez and L. J. Mawst, " $\Gamma$ effect: Key intermodal-discrimination mechanism in arrays of antiguided diode lasers," Appl. Phys. Lett., vol. 60, pp. 3096-3098, 1992. 